to the cells they infect. The transformation of cells grown in tissue culture by viruses or by chemical carcinogens ${ }^{4}$ opens up many new possibilities for research. Hopes are justifiably high that this approach will lead both to a new understanding of carcinogenic mechanisms and to new ways of preventing and possibly treating cancer. It seems, for instance, to be only a matter of time before a virus or group of viruses is found to be causing Burkitt's African lymphoma and methods of preventing its spread in affected communities developed. ${ }^{5}{ }^{6}$ M. H. Salaman and F. J. C. Roe now regard co-carcinogenesis in much broader terms than simply the potentiation of the activity of one chemical carcinogen by that of another. In their review of this subject they discuss carcinogenesis by combinations of chemical, physical, genetic, and viral factors, and it is clear both from the examples they give and from the closing remarks in L. F. Lamerton's article on radiation carcinogenesis that nobody in future can afford to consider any one of these types of factor in isolation.

There is still a wide gap between, on the one hand our knowledge of nucleic acids as building materials and, on the other, the expression of the genetic plan as seen in the fully developed organism. The next few years will see a narrowing of this gap and, we hope, an increased understanding of the mechanisms by which information available within a cell is expressed or remains unexpressed. Meanwhile there are some who suspect that a failure in these mechanisms may sometimes underlie the development of cancer.

\section{Rough Voice}

Some people develop a permanently rough voice. The onset is insidious, though occasionally the patient may date it from an acute infection of the upper respiratory tract. Up to 20 or 30 years ago there could have been several infective causes, chiefly tuberculosis and syphilis, but these are now rarities. Any adult developing a rough voice which lasts a few weeks or months, and who goes to his doctor with this symptom, will almost certainly be referred to a laryngologist at once, for every doctor, and indeed most patients, are nowadays cancer-conscious and recognize the need for early diagnosis. However, a number of patients seem unaware of the disability and if it arises slowly and gradually come to accept it as normal. They may therefore not go to any doctor for years and be impelled to do so only by complete aphonia resulting from a superimposed acute infection. Indeed, some of these people probably never come under medical supervision. When they do, and even though their otherwise good general condition and the long history seem to indicate an innocuous condition, or perhaps a normal variation of personal quality, they should undoubtedly be referred for a specialist examination.

The occurrence of non-specific hypertrophic changes in the larynx has been recognized for many years. French clinicians were foremost in their interest, and J. Garel ${ }^{1}$ described many different types, obtaining material for histological examination by intralaryngeal manœuvres conducted with mirror (indirect) laryngoscopy. The increasing use of direct laryngoscopy popularized by Chevalier Jackson led to more accurate observation of the lesions and more precisely selected biopsy specimens. Most of the epithelium of the larynx is stratified squamous and it is usually non-keratinized. Abnormal activity sometimes occurs in it, as Maxwell Ellis has described, giving rise to hyperplastic changes which seem to form a number of fairly specific clinical patterns with hoarseness as the dominant symptom. ${ }^{2}$ Keratinization in and multiplication of the superficial layers are changes of special interest, as there is some evidence that they may sometimes be precancerous. C. M. Norris and A. R. Peale ${ }^{3}$ have presented histological studies of this reaction, on which they believe prognostic classification can be based. Briefly, when the hyperplastic changes result in crowding and loss of the normal arrangement of layers, and when keratinization occurs in the deeper layers together with nuclear swelling and irregularity, hyperchromatism, and increase in the number of mitotic figures, the condition may progress to carcinoma in situ or even squamous-cell carcinoma. These changes are occasionally confined to one vocal cord and are then very likely indeed to lead to carcinoma. ${ }^{4}$ However, in most cases the lesion is not neoplastic ${ }^{5}$ and responds to treatment.

There is little doubt that the changes in the epithelium are induced by abuse of the voice or by local irritants, chiefly tobacco-smoke, ill-ventilated atmospheres contaminated by chemicals, and excessive alcohol, especially spirits. Constitutional disorders like hypothyroidism and vitamin deficiency may play some part. In all cases of chronic hypertrophic laryngitis some or all of these factors are present, and there must be an as yet undiscovered personal idiosyncrasy which leads to the development of one or another type of lesion. H. Russell ${ }^{6}$ suggested familial peculiarity and an inherited weakness. Abuse of the voice and tobacco are probably the two most important factors in stimulating keratinization. The changes in the larynx can usually readily be seen with the use of the head-mirror, though in some heavy smokers a preliminary local anaesthetic in the oropharynx may be necessary. After the diagnosis has been made clinically, confirmatory direct laryngoscopy and biopsy are essential to establish the type of hyperplasia.

Provided histological examination excludes the possibility of cancer, treatment is primarily a question of management of the patient. The habit of speaking loudly, or shouting, or screaming, must be corrected, and where industrial or other conditions make it necessary to speak above background noise speech therapy can be helpful. Any abuse of tobacco and alcohol must be stopped and indulgence in them is best given up altogether. Where possible the ventilation of stuffy and smoky atmospheres should be improved and constitutional disorders treated. Pine steam inhalations are a helpful sedative at all stages of these conditions. The laryngologist can, however, do something more. Hypertrophic masses can be removed by direct laryngoscopy or even on occasions by laryngofissure. Norris ${ }^{3}$ maintained that with careful operating technique keratotic masses can be stripped off the vocal cords without damaging the deeper layers of the epithelium. Operative intervention of any kind must necessarily be followed by a strict regime embracing the measures discussed above as well as by routine follow-up examinations, because these patients are probably exhibiting a personal reaction to their environment which may recur.

\footnotetext{
1 Garel, J., Monogr. oto-rhino-laryng. int., No. 4, 1921. Paris.

${ }_{2}$ Ellis, M., in Diseases of the Ear, Nose, and Throat, ed. W. G. ScottBrown, 1952. London.

3 Norris, C. M., and Peale, A. R., f. Laryng., 1963, 77, 635.

4 Ellis, M., ibid., 1963, 77, 733.

s Lederman, M., ibid., 1963, 77, 651.

- Russell, H., ibid., 1963, 77, 648.
} 